\title{
PROCESSOS NATURAIS DE BIODEGRADAÇÃO DO PETRÓLEO EM RESERVATÓRIOS
}

\author{
Georgiana Feitosa da Cruz* \\ Laboratório de Engenharia e Exploração de Petróleo, Centro de Ciências e Tecnologia, Universidade Estadual do Norte Fluminense, \\ Rodovia Amaral Peixoto, km 163, 27925-535 Macaé - RJ, Brasil \\ Anita Jocelyne Marsaioli \\ Instituto de Química, Universidade Estadual de Campinas, CP 6154, 13083-862 Campinas - SP, Brasil
}

Recebido em 30/8/11; aceito em 21/3/12; publicado na web em 26/6/12

\begin{abstract}
NATURAL PROCESSES OF PETROLEUM BIODEGRADATION IN RESERVOIRS. Petroleum biodegradation in reservoirs is a process caused by different microorganisms affecting many oil deposits which modifies the oil composition in a quasi-stepwise process starting from $n$-alkanes and isoprenoids through to diasteranes. This causes oil souring and increased viscosity, sulfur and metal content, having a direct impact on oil production and refining costs.
\end{abstract}

Keywords: aerobic and anaerobic microorganisms; petroleum biomarkers; oil composition.

\section{INTRODUÇÃO}

O petróleo, formado a partir do craqueamento térmico do querogênio, é uma mistura complexa de hidrocarbonetos alifáticos, aromáticos e naftênicos, além de outros compostos contendo enxofre, oxigênio, nitrogênio e constituintes organometálicos complexados com níquel e vanádio. ${ }^{1}$ Dentre esses compostos destacam-se os biomarcadores ou fósseis geoquímicos originados de organismos vivos, ${ }^{2-4}$ que são classificados como alcanos lineares, alcanos ramificados, isoprenoides, cicloalcanos, terpanos, esteranos e aromáticos. Os terpanos podem ser bi-, tri-, tetra- e pentacíclicos (hopanos e não hopanoides). O estudo destes compostos é de grande relevância para a indústria do petróleo porque fornecem informações sobre origem, ${ }^{2,5,6}$ ambiente deposicional, ${ }^{3,7,8}$ evolução térmica ${ }^{8-10}$ e índice de biodegradação. ${ }^{2}$

Durante o processo de migração, o petróleo pode sofrer alterações na sua composição devido à geocromatografia, solubilização em água (water washing), mudanças de fase, extração de matéria orgânica presente nas rochas por onde ele passa e/ou biodegradação. ${ }^{11}$ A biodegradação é um processo de alteração do óleo cru por diferentes microrganismos. ${ }^{12,13} \mathrm{~A}$ ocorrência de petróleo biodegradado está vinculada a determinadas condições geológicas e geoquímicas que permitem melhorar a vida microbiana, tais como as existentes na interface óleo-água em um reservatório de petróleo. ${ }^{14}$ De acordo com Huang et al., ${ }^{15}$ a difusão de hidrocarbonetos da coluna de óleo para a zona de contato óleo-água pode controlar e limitar os processos de biodegradação. No início do processo, os hidrocarbonetos são utilizados pelos microrganismos como fonte de energia (doadores de elétrons), enquanto os nutrientes (oxigênio molecular, nitratos, sulfatos ou íon férrico) são necessários como receptores de elétrons para a atividade microbiana. Ao final do processo de biodegradação os hidrocarbonetos são transformados em metabólitos, tais como ácidos orgânicos e/ou $\mathrm{CO}_{2}{ }^{16}$ levando a uma diminuição do teor de hidrocarbonetos saturados e do grau API (American Petroleum Institute) e a um progressivo aumento do conteúdo de asfaltenos, de alguns metais, da densidade, do teor de enxofre, da acidez (formação de ácidos carboxílicos e fenóis) e da viscosidade. Essa alteração nas características do petróleo afeta a produção e eficiência do refino, ${ }^{17}$ aumentando os custos do processo. A Figura 1 apresenta um mecanismo

*e-mail: geofec@gmail.com esquemático de biodegradação dentro de um reservatório de petróleo e as mudanças no grau API ao longo do processo.
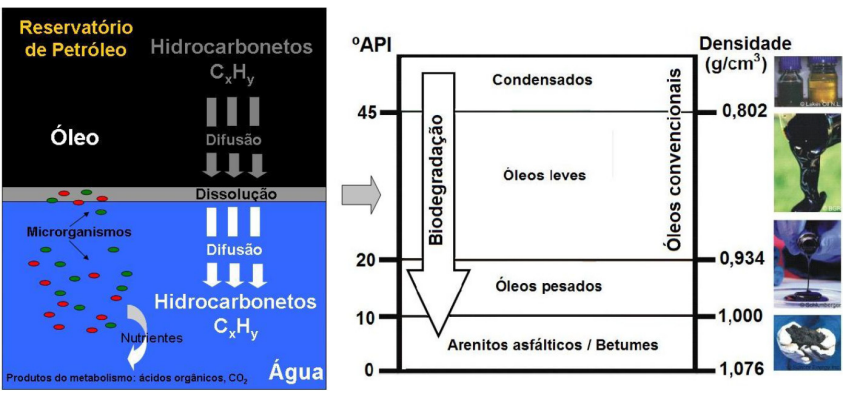

Figura 1. Mecanismo esquemático do processo de biodegradação em um reservatório de petróleo, levando a modificações na sua qualidade com diminuição do grau API. Adaptada da ref. 16

Miiller et al. ${ }^{18}$ encontraram algumas alterações nas propriedades de petróleos de Oklahoma com o aumento dos níveis de biodegradação, como mostra a Tabela 1. As propriedades físico-químicas do petróleo mudam, porque as diferentes classes de compostos que o constitui têm suscetibilidades diferentes à biodegradação. Larter $e t$ al. ${ }^{19}$ estimam que os óleos altamente biodegradados perdem na ordem de $50 \%$ da sua massa inicial.

\section{CONDIÇÕES NECESSÁRIAS PARA OCORRER BIODEGRADAÇÃO}

A maior parte do petróleo do mundo é total ou parcialmente biodegradado em reservatórios e este processo pode levar milhões de anos para ocorrer. ${ }^{20}$ Para se ter um melhor entendimento dos mecanismos de biodegradação do óleo é necessário utilizar condições ideais para que o processo ocorra em curtos intervalos de tempo, quando comparado ao tempo geológico. ${ }^{12,13,21} \mathrm{Em}$ geral, para que ocorra biodegradação de petróleo é necessário: ${ }^{22}$ i) a presença de aceptores de elétrons (ex., oxigênio molecular, nitratos, sulfatos, íons férricos) e nutrientes inorgânicos (ex., fósforo, traço de metais), necessários no início do processo e para manutenção dos microrganismos, respectivamente; ii) a rocha geradora deve ter porosidade e permeabilidade suficientes para permitir a difusão de nutrientes e 
Tabela 1. Mudanças nas propriedades físico-químicas de petróleos com o aumento da biodegradação. Adaptada da ref. 18

\begin{tabular}{ccccccccc}
\hline $\begin{array}{c}\text { Tipos de } \\
\text { petróleo }^{\mathrm{a}}\end{array}$ & API & $\mathrm{S}(\%)$ & $\mathrm{V}(\mathrm{ppm})$ & $\mathrm{Ni}(\mathrm{ppm})$ & $\mathrm{SAT}^{\mathrm{b}}(\%)$ & ARO $(\%)^{\mathrm{c}}$ POLAR $(\%)$ & ASF $^{\mathrm{d}}(\%)$ \\
\hline PNB & 32 & 0,6 & 30,6 & 16,4 & 55 & 23 & 21 & 2 \\
PMB & 12 & 1,6 & 224,0 & 75,1 & 25 & 21 & 39 \\
PP & 4 & 1,5 & 137,5 & 68,5 & 20 & 21 & 41 & 21 \\
\hline
\end{tabular}

${ }^{\text {aPNB }}$ = petróleo não biodegradado; $\mathrm{PMB}=$ petróleo moderadamente biodegradado; $\mathrm{PP}=$ petróleo pesado (arenitos asfálticos); ${ }^{\mathrm{b}} \mathrm{SAT}=$ saturados; ${ }^{\mathrm{c}} \mathrm{ARO}=$ aromáticos; ${ }^{\mathrm{d}} \mathrm{ASF}=$ asfaltenos.

mobilidade de microrganismos. Rochas com baixa porosidade e permeabilidade, com grãos pequenos e sem fraturas, dificultam a difusão de nutrientes e mobilidade microbiana, consequentemente, possuem óleos menos biodegradados; iii) a temperatura do reservatório não deve ultrapassar $\sim 80^{\circ} \mathrm{C}$. Considerando o gradiente geotérmico típico $\left(25-30^{\circ} \mathrm{C} / \mathrm{km}\right)$, essa temperatura é atingida em aproximadamente 2-3 km de profundidade; ${ }^{16}$ iv) a salinidade da água de formação deve estar na faixa de 100-150\%o. ${ }^{20}$ Óleos em reservatórios de petróleo com maior salinidade são tipicamente não biodegradáveis; v) a presença de microrganismos capazes de degradar componentes do petróleo; vi) a ausência de "venenos naturais", isto é, de microrganismos não biodegradadores de hidrocarbonetos, que são tolerantes às condições dos reservatórios e que inibem e/ou limitam o crescimento e atividade enzimática de microrganismos degradadores de petróleo. ${ }^{23}$

Se todas estas condições estão presentes, o petróleo é alterado pelos microrganismos levando a uma diminuição da sua qualidade.

\section{Aceptores de elétrons e nutrientes inorgânicos}

Um dos fatores primordiais para ocorrer biodegradação é a presença de aceptores de elétrons e nutrientes adequados para manter ativos microrganismos de diferentes espécies. Os microrganismos aeróbios necessitam da presença de oxigênio molecular (condições óxicas) para seu crescimento e para converter os hidrocarbonetos em $\mathrm{CO}_{2}, \mathrm{H}_{2} \mathrm{O}$ e biomassa. Já os microrganismos facultativos podem crescer na presença (aerobiose) ou ausência (anaerobiose, fermentação) de oxigênio ${ }^{22,24,25}$ (condições anóxicas). Os anaeróbios estritos utilizam diferentes aceptores, como nitratos $\left(\mathrm{NO}^{3-}\right)$, sulfatos $\left(\mathrm{SO}_{4}{ }^{2-}\right)$ e íons férricos $\left(\mathrm{Fe}^{3+}\right)$ para metabolizar hidrocarbonetos. Outros anaeróbios (aerotolerantes) crescem na presença de concentrações-traços de oxigênio dissolvido, porém não utilizam este gás metabolicamente. ${ }^{25,26}$ Além disso, existem aqueles microrganismos que requerem aceptores de elétrons específicos, como os fermentadores ou acetogênicos que utilizam acetatos $\left(\mathrm{CH}_{3} \mathrm{COO}^{-}\right)$para converter os hidrocarbonetos em $\mathrm{CH}_{4}$ e $\mathrm{CO}_{2} \cdot{ }^{25} \mathrm{~A}$ Tabela 2 mostra alguns microrganismos, seus aceptores de elétrons e os principais produtos gerados a partir da biodegradação de componentes do petróleo. ${ }^{27}$

$\mathrm{Na}$ ausência de aceptores de elétrons, tais como sulfato e nitrato, os hidrocarbonetos são transformados em produtos finais $\left(\mathrm{CH}_{4} \mathrm{e} \mathrm{CO}_{2}\right)$ através da interação de vários grupos de microrganismos como, por exemplo, bactérias fermentativas, acetogênicas produtoras de hidrogênio, metanogênicas hidrogenotróficas e acetoclásticas (Figura 4). ${ }^{28,29}$

Nutrientes inorgânicos, tais como nitrogênio $(\mathrm{N})$ e fósforo $(\mathrm{P})$, são essenciais na biossíntese de proteínas, ácidos nucleicos e fosfolipídios e traços de metais, como molibdênio (Mo), cobalto $(\mathrm{Co})$ e cobre $(\mathrm{Cu})$, são importantes em reações enzimáticas. Alguns desses nutrientes são incorporados na matriz da rocha geradora de petróleo durante a diagênese (degradação bioquímica ou térmica da matéria orgânica para gerar petróleo) ou através de alteração secundária (water washing ou biodegradação) e migram para a rocha reservatório tornando-se um dos seus componentes. ${ }^{30,31}$ Tanto os nutrientes inorgânicos, quanto alguns metais podem limitar o processo de biodegradação do petróleo. ${ }^{21}$
Tabela 2. Aceptores de elétrons utilizados por diferentes microrganismos na biodegradação de hidrocarbonetos. Adaptada da ref. 27

\begin{tabular}{lcc}
\hline Microrganismos & $\begin{array}{c}\text { Aceptores de } \\
\text { elétrons }\end{array}$ & Produtos \\
\hline Aeróbios & $\mathrm{O}_{2}$ & $\mathrm{CO}_{2}$ \\
Metanogênicos & - & $\mathrm{CH}_{4} \mathrm{e} \mathrm{CO}_{2}$ \\
Metanogênicos & $\mathrm{CO}_{2}$ & $\mathrm{CH}_{4}$ \\
BRS & $\mathrm{SO}_{4}{ }^{2-}$ & $\mathrm{H}_{2} \mathrm{~S}$ \\
$\begin{array}{l}\text { Denitrificantes (Veillonella alcalescens, } \\
\text { Selenomonas ruminantium, Clostridium } \\
\text { perfringens) }\end{array}$ & $\mathrm{NO}_{3}^{-}$ & $\mathrm{NO}_{2}^{-}$ \\
$\begin{array}{l}\text { Denitrificantes (Propionibacterium } \\
\text { pentosaceum) }\end{array}$ & $\mathrm{NO}_{2}^{-}$ & \\
Outros anaeróbios & & $\mathrm{N}_{2}$ \\
\hline
\end{tabular}

\section{Influência da temperatura e pressão na biodegradação do petróleo}

A temperatura ótima para ocorrer biodegradação de petróleo no reservatório é aproximadamente $60-80^{\circ} \mathrm{C},{ }^{22,32}$ entretanto, simulações laboratoriais indicam que a biodegradação de hidrocarbonetos ocorre pela ação de microrganismos que crescem a diferentes temperaturas, tais como, psicrofílicos $\left(0-25^{\circ} \mathrm{C}\right)$, mesofílicos $\left(30-40^{\circ} \mathrm{C}\right)$ e termofíli$\cos \left(50-60^{\circ} \mathrm{C}\right) .{ }^{25}$ Walker e Colwell ${ }^{33}$ demonstraram a biodegradação de alcanos por microrganismos obtidos a partir de água de formação e sedimentos, na faixa de temperatura entre $0-10{ }^{\circ} \mathrm{C}$, enquanto Rueter $e t$ $a l .{ }^{34}$ isolaram uma BRS termofílica que biodegrada alcano em temperaturas acima de $60^{\circ} \mathrm{C}$. A influência da temperatura na biodegradação de hidrocarbonetos depende, portanto, do tipo de microrganismo e das condições utilizadas no processo.

Vale ressaltar que nem todas as acumulaçãoes de óleo em temperaturas inferiores a $80{ }^{\circ} \mathrm{C}$ são biodegradadas. Wilhelms et al. ${ }^{35,36}$ sugeriram que, se um reservatório de óleo foi aquecido a mais de 80 ${ }^{\circ} \mathrm{C}$ em qualquer momento desde sua deposição, ocorrerá a "paleopasteurização" ou "esterilização" do óleo, provocando a mortandade dos microrganismos biodegradadores, consequentemente, mesmo que a temperatura seja novamente inferior a $80^{\circ} \mathrm{C}$, o petróleo não será biodegradado. Portanto, reservatórios de óleo que sofreram elevação significativa da temperatura podem conter petróleo não biodegradado, mesmo possuindo baixas profundidade e temperatura, visto que a "recolonização" por bactérias do óleo "esterilizado" no reservatório é tipicamente incapaz de ocorrer.

Ao contrário da temperatura, a pressão do reservatório não é um fator limitante para o crescimento microbiano. ${ }^{22}$ Moldowan et al. ${ }^{37}$ analisaram óleos biodegradados em reservatórios a $2500 \mathrm{~m}$ na Bacia Adriática Central (Itália e Iugoslávia), enquanto Walters ${ }^{38}$ estudou óleos altamente biodegradados em reservatórios de águas profundas a 4000 m no Sul da Bacia do Mar Cáspio (Azerbaijão). Em ambos os casos, a biodegradação ocorreu com geopressões diferentes e temperaturas inferiores a $80{ }^{\circ} \mathrm{C}$. 


\section{Microrganismos envolvidos no processo de biodegradação do petróleo}

Desde o início da produção comercial de petróleo, os geoquímicos têm se deparado com problemas relacionados à presença de microrganismos nos reservatórios. ${ }^{39}$ Os microrganismos, além de consumirem os hidrocarbonetos, são também citados como os grandes responsáveis pela corrosão de equipamentos e tanques de estocagem, devido aos subprodutos originados da biodegradação. ${ }^{40}$

Os microrganismos envolvidos no processo de biodegradação ainda são objetos de discussões conflitantes, uma vez que se atribui a degradação tanto aos aeróbios, ${ }^{39-41}$ quanto aos anaeróbios. ${ }^{42-44}$ Aqueles que defendem a ação dos microrganismos aeróbios (condições óxicas) assumem que a temperatura durante o processo está entre $60-80{ }^{\circ} \mathrm{C}$ e envolve a incursão de águas meteóricas que carreia oxigênio e nutrientes necessários à manutenção destes microrganismos dentro do reservatório, ${ }^{32}$ enquanto aqueles favoráveis à degradação anaeróbia (condições anóxicas) indicam que a demanda de oxigênio no reservatório é insuficiente para manter os aeróbios ativos e, portanto, os microrganismos que degradam o petróleo são estritamente anaeróbios, degradando os componentes do petróleo mais lentamente. ${ }^{36,44,45}$

No entanto, existem evidências científicas que indicam que estes dois grupos de microrganismos biodegradam o petróleo através de ação sinérgica em tempos geológicos diferentes, onde um complementa o outro sem sobreposição. ${ }^{23,24,46}$ Os componentes do petróleo e outros metabólitos que são recalcitrantes para os aeróbios presentes no reservatório são facilmente biodegradados pelos anaeróbios e, por outro lado, aqueles que são de difícil degradação pelos anaeróbios podem ser completamente biodegradados pelos aeróbios. ${ }^{23,24}$

\section{MUDANÇAS NA COMPOSIÇÃO QUÍMICA DO PETRÓLEO}

\section{$n$-alcanos e compostos aromáticos}

Conhece-se da literatura que a biodegradação microbiana afeta significativamente a composição molecular e as propriedades físicas do petróleo bruto, levando a uma diminuição dos compostos de baixo peso molecular, presentes nas frações saturada e aromática, e a um aumento da fração polar (fração asfaltênica) ${ }^{18,21,22,46-49}$ (Figura 1 e Tabela 1). Essas mudanças ocorrem devido à remoção quase sequencial e sistemática dos compostos presentes no petróleo, iniciando pelos hidrocarbonetos mais leves $\left(\mathrm{C}_{6} \mathrm{a}_{15}\right)$ até aqueles mais resistentes ao ataque microbiano (ex.: diasteranos, dia-hopanos). ${ }^{24}$

Os hidrocarbonetos são preferencialmente removidos durante a biodegradação inicial, mas compostos contendo enxofre (tióis, dissulfetos, tioalcanos), nitrogênio (carbazóis) e oxigênio (furanos) também podem ser biodegradados, gerando novos compostos como ácidos saturados, aromáticos, cíclicos e acíclicos e fenóis. ${ }^{20}$

A microbiota presente nos reservatórios de petróleo possui capacidade enzimática e preferências metabólicas diferenciadas para degradar esses componentes. Uns degradam preferencialmente alcanos normais, ramificados ou cíclicos, outros preferem mono ou poliaromáticos e existem ainda aqueles que degradam tanto alcanos quanto aromáticos. ${ }^{14}$

A principal via metabólica de biodegradação de hidrocarbonetos por microrganismos aeróbios já está bem estabelecida na literatura e pode ocorrer na porção terminal e/ou subterminal da cadeia carbônica. ${ }^{25,50,51}$ Os $n$-Alcanos de cadeias longas $\left(\mathrm{C}_{10}-\mathrm{C}_{24}\right)$ são mais rapidamente degradados a partir da porção terminal, enquanto os $n$-alcanos de cadeias menores que $\mathrm{C}_{10}$ são tóxicos para a maioria dos microrganismos e sua oxidação é iniciada pela porção subterminal da cadeia.
A etapa inicial do processo de biodegradação é a oxidação do substrato por oxigenases, com a utilização de oxigênio molecular como aceptor de elétrons. A oxidação terminal inicia-se com a formação de um álcool primário. Após a etapa inicial, o álcool é oxidado, por ação das enzimas álcool e aldeído desidrogenases, aos correspondentes aldeído e ácido carboxílico, respectivamente. O ácido carboxílico, por sua vez, é utilizado como substrato para acil-CoA sintetase e é posteriormente biodegradado pelo processo de $\beta$-oxidação. Os $n$-alcanos de cadeias menores são oxidados subterminalmente $\left(1^{\circ}\right.$ carbono secundário da cadeia do $n$-alcano), formando álcool secundário, cetona, éster e ácidos carboxílicos, que são posteriormente biodegradados pelo processo de $\beta$-oxidação (Figura 2).

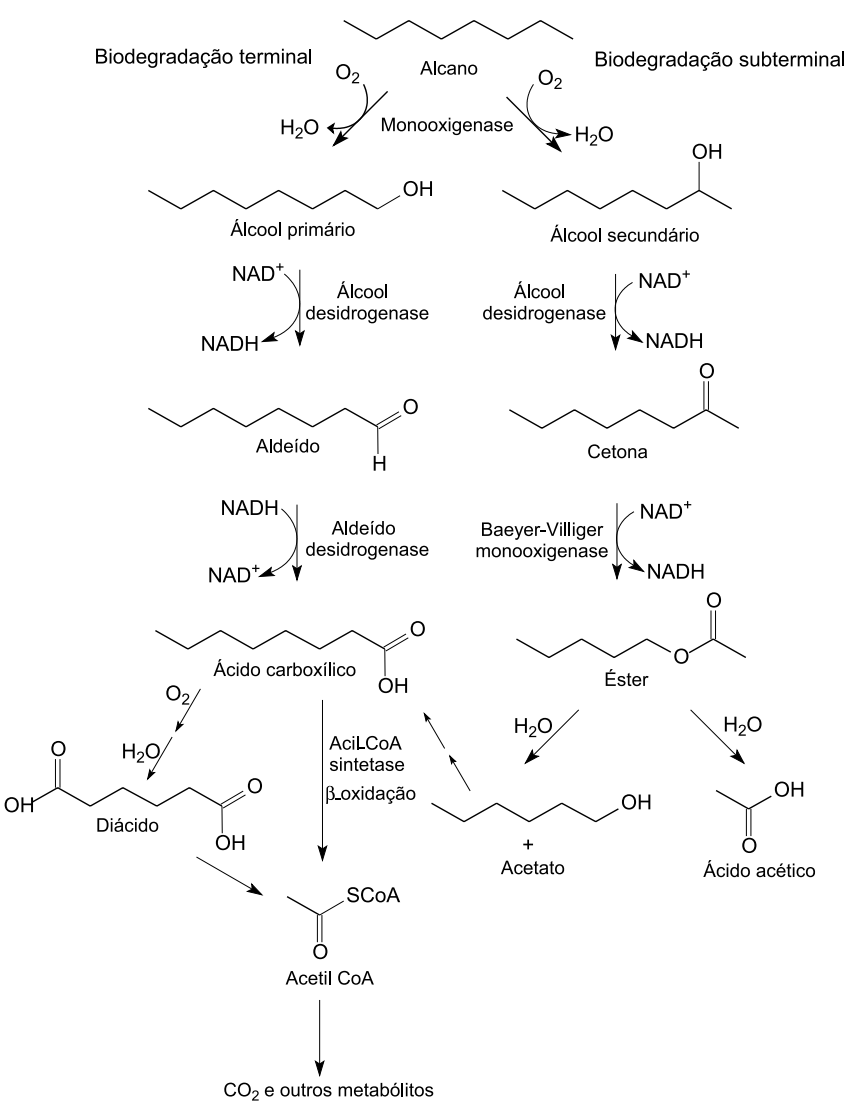

Figura 2. Rotas metabólicas de biodegradação aeróbia de n-alcanos. Adaptada da ref. 57

A biodegradação de compostos aromáticos por microrganismos aeróbios também é iniciada pela ação de oxigenases (mono- ou dioxigenases) que promovem a oxidação do substrato formando dióis, seguida de clivagem do anel e formação de catecol que, posteriormente, é degradado formando diferentes intermediários, como

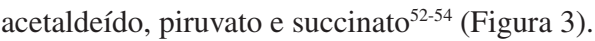

O conhecimento das rotas de biodegradação para anaeróbios, no entanto, é limitado. Sob condições anóxicas, os compostos orgânicos são sucessivamente oxidados, ou seja, os produtos de cada etapa de oxidação atuam como substratos nas etapas seguintes, até que a matéria orgânica seja completamente degradada a biogás. ${ }^{55}$

Existem vários relatos sobre a oxidação de hidrocarbonetos com culturas puras de bactérias redutoras de sulfato (BRS) em condições estritamente anaeróbias, ${ }^{34,56-58}$ denitrificantes ${ }^{59}$ e metanogênicas. ${ }^{60}$ As BRS e denitrificantes utilizam sulfatos e nitratos, respectivamente, como aceptores de elétrons para biodegradar hidrocarbonetos, análogo ao oxigênio molecular na biodegradação aeróbia. ${ }^{51}$ Já as bactérias metanogênicas metabolizam hidrocarbonetos ou na ausência de 


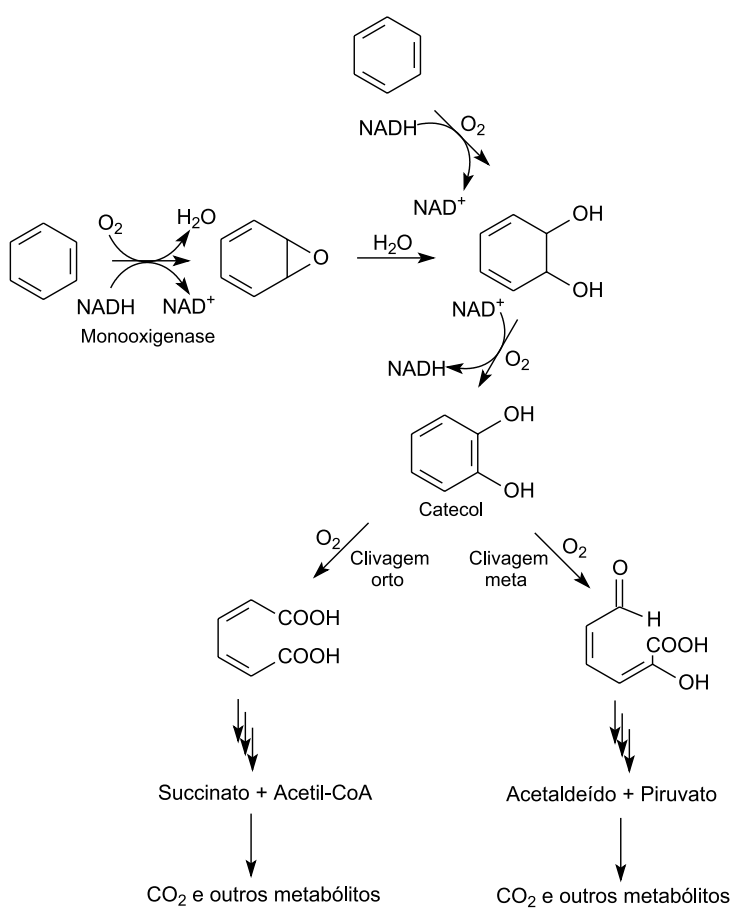

Figura 3. Rota metabólica de biodegradação aeróbia de hidrocarbonetos aromáticos. Adaptada da ref. 57

aceptores de elétrons, convertendo-os a metano e $\mathrm{CO}_{2}$, ou na presença de $\mathrm{CO}_{2}$, convertendo os hidrocarbonetos para $\mathrm{CH}_{4} \cdot{ }^{61}$ Existem, ainda, as bactérias anaeróbias acetogênicas que consomem os hidrocarbonetos gerando acetato e $\mathrm{H}_{2}^{62}$ (Figura 4).

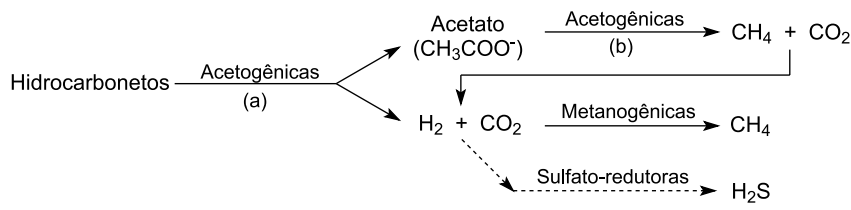

(a) $4 \mathrm{C}_{16} \mathrm{H}_{34}+6 \mathrm{H}_{2} \mathrm{O} \longrightarrow 32 \mathrm{CH}_{3} \mathrm{COO}^{-}+32 \mathrm{H}^{+}+68 \mathrm{H}_{2}$

(b) $32 \mathrm{CH}_{3} \mathrm{COO}^{-}+32 \mathrm{H}^{+} \longrightarrow 32 \mathrm{CH}_{4}+32 \mathrm{CO}_{2}$

Figura 4. Caminho de degradação de hidrocarbonetos a $\mathrm{CH}_{4}, \mathrm{CO}_{2}$ e $\mathrm{H}_{2} \mathrm{~S} \mathrm{por}$ microrganismos anaeróbios. Adaptada da ref. 63

Os mecanismos de biodegradação anaeróbia de hidrocarbonetos utilizando consórcios de microrganismos e bactérias isoladas são bastante estudados, porém pouco entendidos. Em geral, dois mecanismos de ativação, tanto para alcanos quanto para aromáticos, têm sido propostos: o primeiro envolve a ativação do carbono subterminal do hidrocarboneto e adição de fumarato, enquanto que o segundo envolve a carboxilação do hidrocarboneto na posição C-3. ${ }^{22,45,51,57,62,63}$ Ambos os caminhos podem ocorrer simultaneamente em consórcios de BRS (Figura 5).

Quando a biodegradação ocorre através do primeiro mecanismo de ativação, o hidrocarboneto é adicionado à dupla ligação do fumarato via átomo de carbono subterminal, gerando ácidos graxos lineares e ramificados e succinatos substituídos. ${ }^{64,65}$ Os ácidos graxos lineares gerados com números pares de carbono provêm de $n$-alcanos também com números pares de carbono e os ácidos graxos com números de carbono ímpares são gerados a partir de $n$-alcanos com números ímpares de carbono. No segundo mecanismo ocorre formação apenas de ácidos graxos lineares, porém os $n$-alcanos pares geram os ácidos graxos ímpares e vice-versa (Figura 5).

A Tabela 3 apresenta algumas bactérias com metabolismo aeróbio e anaeróbio, que são capazes de utilizar hidrocarbonetos como fonte de carbono.

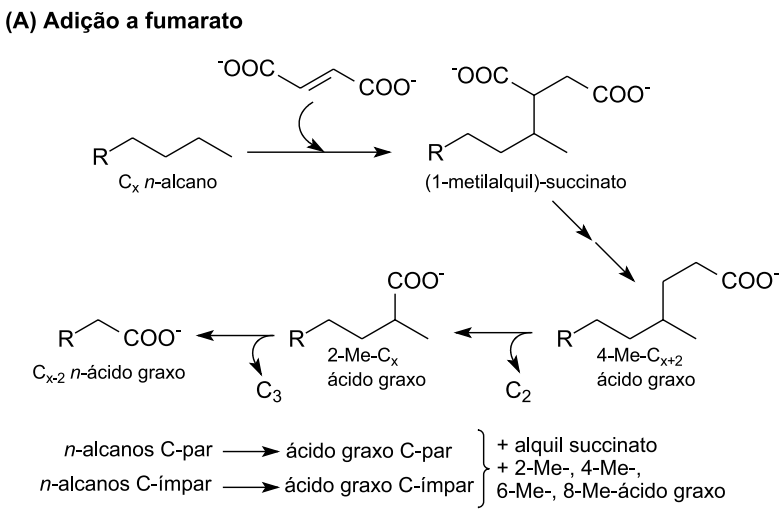

(B) Carboxilação a C-3

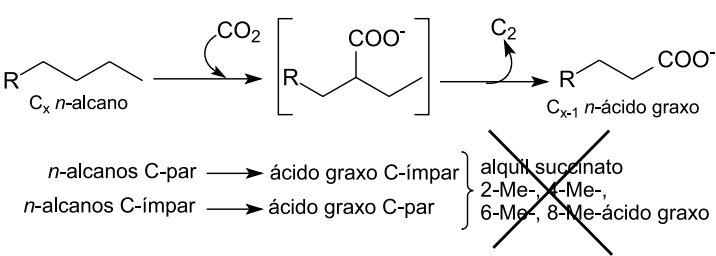

Figura 5. Rota de biodegradação anaeróbia para hidrocarbonetos via fumarato e carboxilação em C-3. Adaptada da ref. 68

Tabela 3. Bactérias aeróbias e anaeróbias que utilizam hidrocarbonetos como fonte de carbono. Adaptada das refs. 1 e 54

\begin{tabular}{|c|c|c|}
\hline Bactéria & Hidrocarboneto & $\begin{array}{c}\text { Tipo de } \\
\text { metabolismo }\end{array}$ \\
\hline Acinetobacter calcoaceticus EB104 & $\mathrm{C}_{9}-\mathrm{C}_{18}$ & Aeróbio \\
\hline Acinetobacter sp. M-1 & $\mathrm{C}_{13}-\mathrm{C}_{44}$ & Aeróbio \\
\hline Alcaligenes odorans $\mathrm{P} 20$ & $-\mathrm{C}_{33}$ & Aeróbio \\
\hline Antrobacter nicotianae $\mathrm{KCC} \mathrm{B} 35$ & $\mathrm{C}_{10}-\mathrm{C}_{40}$ & Aeróbio \\
\hline Bacillus thuringiensis/cereus A2 & $\mathrm{C}_{6}-\mathrm{C}_{28}$ & Aeróbio \\
\hline Dietzia psychralcaliphila & $\mathrm{C}_{13}-\mathrm{C}_{24}$ & Aeróbio \\
\hline Marinobacter sp. BC36 & $-\mathrm{C}_{18}$ & Aeróbio \\
\hline Mycobacterium sp. PYR-1 & Antraceno & Aeróbio \\
\hline Mycobacterium sp. CH1 & $\mathrm{C}_{12}-\mathrm{C}_{28}$ & Aeróbio \\
\hline Nocarsiodes sp. KP7 & Fenantreno & Aeróbio \\
\hline Pseudomonas aeruginosa $\mathrm{PAO} 1$ & $\mathrm{C}_{12}-\mathrm{C}_{24}$ & Aeróbio \\
\hline Pseudomonas sp. U2 & Naftaleno & Aeróbio \\
\hline Rhodococcus sp.1BN & $\mathrm{C}_{6}-\mathrm{C}_{28}$ & Aeróbio \\
\hline Rhodococcus sp. 124 & Tolueno & Aeróbio \\
\hline Thermooleophilum album & $\mathrm{C}_{13}-\mathrm{C}_{20}$ & Aeróbio \\
\hline Xylella fastidiosa RR15 & $\mathrm{C}_{14}-\mathrm{C}_{34}$ & Aeróbio \\
\hline Azoarcus sp. EB1 & Etilbenzeno & Denitrificante \\
\hline Dechloromassp. JJ & Benzeno, tolueno & Denitrificante \\
\hline Cepa OcN1 & $\mathrm{C}_{8}-\mathrm{C}_{12}$ & Denitrificante \\
\hline Marinobacter sp. BC36 & $-\mathrm{C}_{18}$ & Denitrificante \\
\hline Pseudomonas sp. NAP-3 & Naftaleno & Denitrificante \\
\hline Marinobacter sp. BC42 & $\mathrm{C}_{6}-\mathrm{C}_{15}$ & Denitrificante \\
\hline Cepa TD3 & $\mathrm{C}_{8}-\mathrm{C}_{12}$ & Sulfatorredutor \\
\hline Cepa Hxd3 & $\mathrm{C}_{12}-\mathrm{C}_{20}$ & Sulfatorredutor \\
\hline Cepa Pnd3 & $\mathrm{C}_{14}-\mathrm{C}_{17}$ & Sulfatorredutor \\
\hline Desulfobacterium cetonicum & Tolueno & Sulfatorredutor \\
\hline Clone B1-B3 & $-\mathrm{C}_{16}$ & Metanogênico \\
\hline
\end{tabular}




\section{Compostos biomarcadores}

Os biomarcadores mais comumente analisados no petróleo pertencem às classes dos terpanos tricíclicos, pentacíclicos (hopanos) e dos esteranos.

Em reservatórios, os terpanos tricíclicos (TT) $\mathrm{C}_{19}-\mathrm{C}_{45}$ são altamente resistentes à biodegradação, removidos após os hopanos ${ }^{66} \mathrm{e}$ ao mesmo tempo em que os diasteranos, ${ }^{67}$ porém, algumas exceções foram observadas utilizando experimentos em laboratório com culturas aeróbias. ${ }^{23,24}$ Estudos recentes reportaram a remoção de TT antes de hopanos, com degradação preferencial de ambos os epímeros $R \mathrm{e}$ $S$ para TT $\mathrm{C}_{28}$ em relação ao TT $\mathrm{C}_{29} \cdot{ }^{24,68,69}$

O mecanismo de degradação de hopanos, 25-nor-hopanos e esteranos, tanto em reservatórios quanto em escala laboratorial, envolve muitas discussões. Alguns estudos com óleos severamente biodegradados no reservatório mostram que os hopanos são removidos antes dos esteranos, com formação de 25-nor-hopanos. ${ }^{70}$ Por outro lado, existem alguns relatos, também com óleos severamente biodegradados no reservatório, indicando que os hopanos são removidos após a quase total degradação dos esteranos, sem formação de 25-nor-hopanos. ${ }^{3}$ Como exemplos, óleos do oeste da Sibéria, severamente biodegradados, mostraram degradação de $17 \alpha(\mathrm{H})$-hopanos com enriquecimento de 25-nor-hopanos e sem degradação de esteranos. ${ }^{71}$ Existem, também, exemplos de petróleos onde a degradação de hopanos e esteranos ocorreu simultaneamente, como é o caso das amostras de asfaltos de Malagasy que apresentaram $17 \alpha(\mathrm{H})$-hopanos, 25-nor-hopanos e esteranos parcialmente degradados..$^{72}$ Em escala laboratorial, a suscetibilidade de biodegradação destes biomarcadores depende das condições do experimento e, também, da população microbiana utilizada. ${ }^{23,24,44}$ Por exemplo, sob condições aeróbias, Bost et al ${ }^{69}$ mostrou que os hopanos são degradados sem produção de 25-nor-hopanos ou degradação de esteranos.

Existe uma grande quantidade de microrganismos descritos na literatura que possuem capacidade enzimática para biodegradar biomarcadores do petróleo. ${ }^{24,76}$ Dentre estes, citam-se, como exemplos, os aeróbios pertencentes aos gêneros Achromobacter, Bacillus, Brevibacterium, Mezorhizobium e Bordetella, descritos na literatura como degradadores de hidrocarbonetos e/ou associados a ambientes contaminados com óleo ${ }^{73-75}$ e alguns anaeróbios facultativos pertencentes aos gêneros Bacillus e Acinetobacter, dentre outros, conhecidos pela capacidade de utilizar hidrocarbonetos como fonte de carbono diretamente ${ }^{76}$ ou por utilizarem os metabólitos produzidos por outras bactérias, quando estão em consórcios. ${ }^{77}$

Um dos principais produtos gerados a partir da biodegradação dos terpanos tricíclicos, hopanos e esteranos são seus correspondentes ácidos terpanoicos tricíclicos, hopanoicos e esteranoicos. ${ }^{78,79}$ Esses ácidos são comumente derivatizados aos correspondentes ésteres metílicos e reduzidos a hidrocarbonetos, para serem analisados por cromatografia gasosa acoplada à espectrometria de massas. A Figura 6 apresenta alguns desses ácidos já detectados em amostras de petróleo.

Os estudos dos componentes ácidos correlacionados com os da fração neutra em amostras de óleos e sedimentos é um campo vasto não totalmente explorado e pode ser utilizado para um melhor entendimento dos processos que envolvem geração, migração e biodegradação dessas amostras. ${ }^{80}$ Óleos crus imaturos, óleos biodegradados, frações e óleos pesados, assim como águas geradas na extração do óleo cru e betume, provenientes de várias partes do mundo, frequentemente apresentam ácidos orgânicos de ocorrência natural em sua constituição. ${ }^{81,82}$

No Brasil, o aparecimento de petróleos ácidos está relacionado com a produção de Cherne e Pampo, na Bacia de Campos ${ }^{83}$ Este surgimento mantém uma estreita relação com a evolução das descobertas e produção de óleo no país.

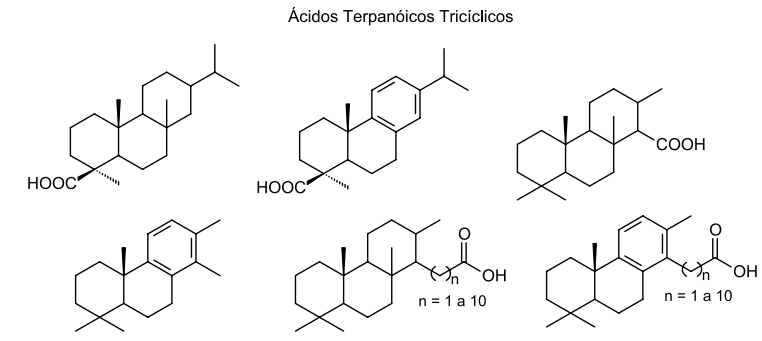

Ácidos Hopanóicos

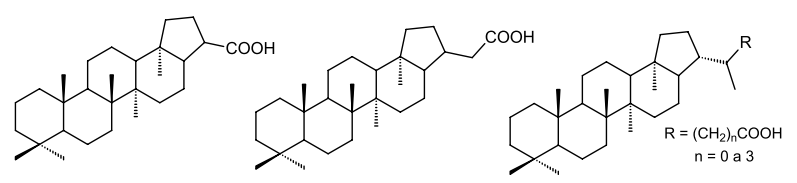

Ácidos Esteranóicos
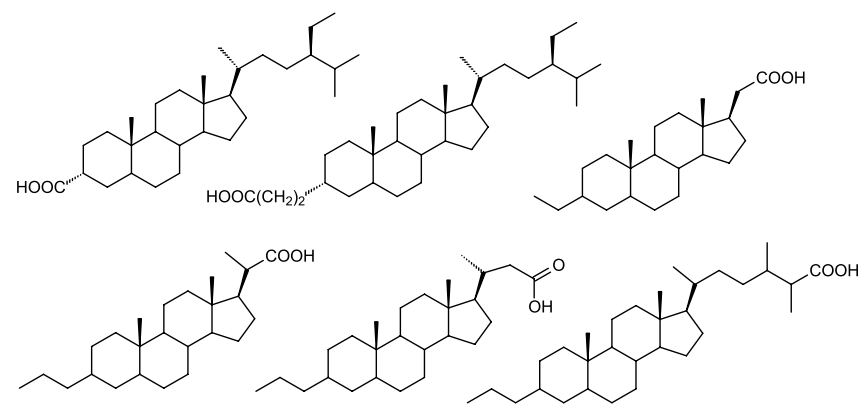

Figura 6. Biomarcadores ácidos detectados em amostras de petróleo formados durante o processo de biodegradação. Adaptada das refs. 79 e 80

\section{AVALIAÇÃO QUALITATIVA DA BIODEGRADAÇÃO - ESCALAS DE BIODEGRADAÇÃO}

Através da análise por cromatografia gasosa é possível acompanhar as mudanças composicionais que ocorrem com as diferentes frações do petróleo. Para isso, realiza-se uma varredura de todos os íons presentes na amostra (Total Ion Chromatogram - TIC) e monitoram-se seletivamente alguns íons característicos das principais classes de biomarcadores (Reconstructed Ion Chromatogram - RIC e Single Ion Monitoring - SIM). ${ }^{22}$

O perfil cromatográfico de uma amostra de petróleo constitui sua impressão digital e é uma das primeiras indicações qualitativas da ocorrência de biodegradação, visto que os compostos lineares são os mais abundantes em óleos não biodegradados e, por isso, os primeiros a serem consumidos pelos microrganismos. Desta forma, quando o perfil da amostra revela diminuição da concentração destes compostos, diz-se que o petróleo sofreu biodegradação tornando-se gradativamente mais pesado e mais ácido.

A extensão do nível de biodegradação em uma amostra de petróleo é medida utilizando-se parâmetros baseados na abundância relativa de várias classes de compostos (n-alcanos, hidrocarbonetos aromáticos, biomarcadores), através da utilização de escalas de biodegradação. Estas escalas mostram o efeito da remoção seletiva dos compostos e classificam o petróleo em níveis que variam de 0 (óleo não biodegradado) a 10 (óleo severamente biodegradado). ${ }^{3,84-89}$ A Figura 7 apresenta uma escala de biodegradação baseada na remoção de grupos moleculares selecionados com o progresso da biodegradação. ${ }^{20,86,87}$ Os compostos encontrados no petróleo são removidos preferencialmente na sequência: $n$-alcanos, alquilcicloexanos, isoprenoides acíclicos, alcanos bicíclicos, esteranos e hopanos, com produção de novos compostos como produtos de biodegradação, tais como 


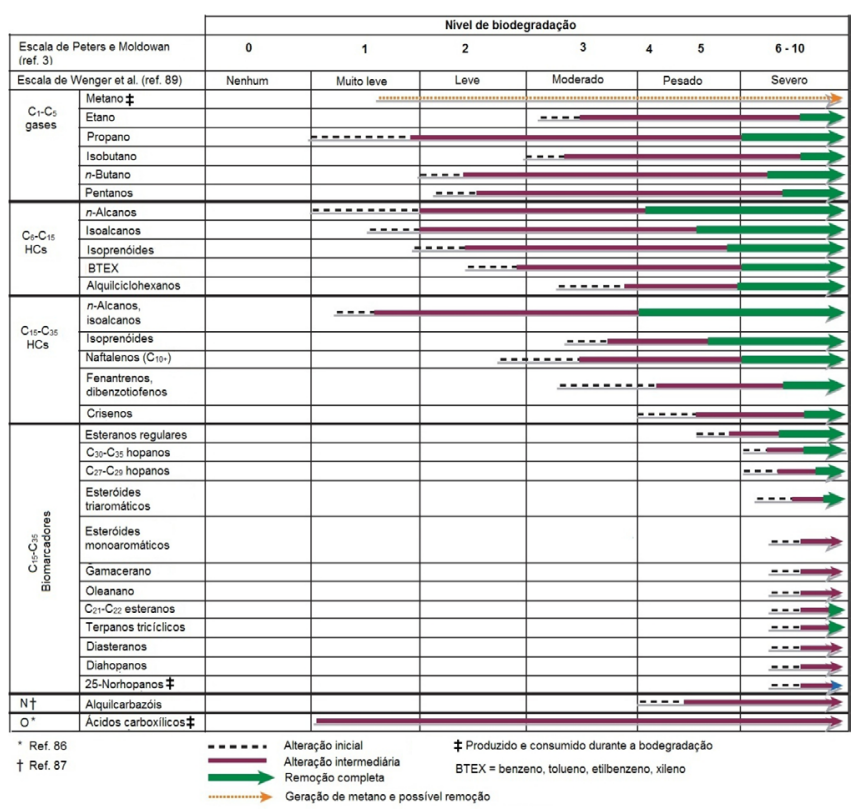

Figura 7. Escala de biodegradação mostrando a remoção seletiva de grupos moleculares com o aumento da biodegradação. Adaptada da ref. 20

17 $\alpha, 25$-nor-hopanos a partir da desmetilação de hopanos em níveis severos de degradação. ${ }^{20,38}$

\section{CONCLUSÃO}

Em condições adequadas de temperatura (até cerca de $80{ }^{\circ} \mathrm{C}$ ), nutrientes inorgânicos e aceptores de elétrons, o petróleo em reservatórios é muitas vezes degradado biologicamente, em escalas de tempo geológico, por microrganismos que destroem hidrocarbonetos e outros componentes, produzindo novos compostos que modificam as propriedades físicas do óleo transformando-o em "óleo pesado", diminuindo seu valor econômico. Como a maior parte do petróleo do mundo é biodegradado, alternativas para a recuperação desses “óleos pesados" estão sendo continuamente avaliadas.

\section{REFERÊNCIAS}

1. van Hamme, J. D.; Singh, A.; Ward, O. P.; Microbiol. Mol. Biol. Rev. 2003, 67, 503 .

2. Aquino Neto, F. R.; Quim. Nova 1984, 7, 79.

3. Peters, K. E.; Moldowan, J. M.; The Biomarker Guide: Interpreting molecular fossil in petroleum and ancient sediments. Prentice Hall Englewood Clifts: New Jersey, 1993.

4. Holba, A. G.; Dzou, L.; Org. Geochem. 2004, 34, 441.

5. Restlé, A.; O Petróleo, tradução e adaptação: Mello, M. R.; Hessel, M. A.; Soldan, A. L.; Petrobrás/CENPES: Rio de Janeiro, 1994.

6. Brocks, J. J.; Buick, R.; Summons, R. E.; Logan, G. A.; Geochim. Cosmochim. Acta 2003, 67, 4321.

7. Philp, R. P.; Fossil Fuel Biomarkers: Applications and Spectra, Elsevier: New York, 1985.

8. Brocks, J. J.; Love, G. D.; Snape, C. E.; Logan, G. A.; Summons, R. E.; Buick, R.; Geochim. Cosmochim. Acta 2003, 67, 1521.

9. Tissot, B. P.; Welte, D. H.; Petroleum Formation and Occurrence, $2^{\text {nd }}$ ed., Springer-Verlag, Berlim Heidelberg: New York, 1978, chap. 5.

10. Killops, S. D.; Killops, V. J.; An Introduction to Organic Geochemistry, $1^{\text {st }}$ ed., John Wiley \& Sons, Inc.: New York, 1993, chap. 5.

11. Trindade, L. A. F.; Dissertation for the degree of Doctor of Philosophy, Stanford University, EUA, 1992.

12. Palmer, S. E. Em Effect of biodegradation and water washing on crude oil composition; Engel, M. H.; Macko, S. A., eds.; Plenum Press: New York, 1993, p. 511.

13. Blanc, P.; Connan, J. Em The Petroleum System - From Source to Trap; Magoon, L. B.; Dow, W. G., eds.; Tulsa: Oklahoma, 1994, p. 237.

14. Larter, S.; Huang, H.; Adams, J.; Bennett, B.; Jokanola, O.; Oldenburg, T. B. P.; Jones, M.; Head, I.; Riediger, C.; Fowler, M. G.; AAPG Bull. 2006, 90, 921.

15. Huang, H.; Bowler, B. F. J.; Oldenburg, T. B. P.; Larter, S. R.; Org. Geochem. 2004, 35, 1619

16. Rouven, E.; Doktor der Naturwissenschaften, Technischen Universität Berlin, Germany, 2008.

17. White, N.; Thompson, M.; Barwise, T.; Nature 2003, 426, 334.

18. Miiller, D. E.; Holba, A. G.; Huges, W. B.; AAPG Studies in Geology 1987, 233

19. Larter, S. R.; Head, I. M.; Huang, H.; Bennett, B.; Jones, M.; Aplin, A. C.; Murray, A.; Erdmann, M.; Wilhelms, A.; di Primio, R. Em Biodegradation, gas destruction and methane generation in deep subsurface petroleum reservoirs: an overview; Dore, A. G.; Vining, B., eds.; Petroleum Geology: Northwest Europe and global perspectives: Proceedings of the $6^{\text {th }}$ Petroleum Geology Conference Series, Geological Society: London, 2005, vol. 6, p. 633.

20. Head, I. M.; Jones, D. M.; Larter, S. R.; Nature 2003, 426, 344.

21. Connan, J. Em Advances in Petroleum Geochemistr; Brooks, J.; Weltes, D. H., eds.; Academic Press: London, 1984, p. 299.

22. Peters, K. E.; Walters, C. C.; Moldowan, J. M.; The Biomarker Guide: Biomarkers and Isotopes in the Environment and Human History, $2^{\text {nd }}$ ed., Cambridge University Press: United Kington, 2005, chap. 6.

23. Da Cruz, G. F.; Tese de Doutorado, Universidade Estadual de Campinas, Brasil, 2009.

24. Da Cruz, G. F.; de Vasconcelos, S. P.; Angolini, C. F. F.; Dellagnezze, B. M.; Garcia, I. N. S.; de Oliveira, V. M.; Santos Neto, E. V.; Marsaioli, A. J.; AMB Express. 2011, 1, 47.

25. Berthe-Corti, L.; Fetzner, S.; Acta Biotechnol. 2002, 3-4, 299.

26. Madigan, M. T.; Martinko, J. M.; Parker, J.; Brock Biology of Microorganisms, $8^{\text {th }}$ ed., Prentice-Hall: New Jersey, 1997, p. 606.

27. Holland, K. T.; Knapp, J. S.; Shoesmith, J. G.; Anaerobic Bacteria, $1^{\text {st }}$ ed., Blackie \& Son: New York, 1987.

28. Colleran, E.; Finnegan, S.; Lens, P.; Antonie Leeuwenhoec. 1995, 67, 29.

29. Oude-Elferink, S. J. W. H.; Rinia, H. A.; Bruins, M. E.; de Vos, W. M.; Stams, A. J. M.; J. Appl. Microbiol. 1997, 83, 102.

30. Holloway, J. M.; Dahlgren, R. A.; Casey, W. H.; Chem. Geol. 2001, 174, 403.

31. da Silva, L. I. D.; Carneiro, M. C.; Emídio, V. dos S.; Henrique Jr., S. de S.; Monteiro, M. I. C.; Quim. Nova 2006, 29, 46.

32. Evans, P. J.; Ling, W.; Goldschmidt, B.; Ritter, E. R.; Young, L. Y.; Appl. Environ. Microbiol. 1992, 58, 496.

33. Walker, J. D.; Cowell, R. R.; Appl. Environ. Microbiol. 1976, 31, 198.

34. Rueter, P.; Rabus, R.; Wilkes, H.; Aeckersberg, F.; Rainey, F. A.; Jannasch, H. W.; Widdel, F.; Nature 1994, 372, 455.

35. Wilhelms, A.; Larter, S. R.; Head, I.; Farrimond, P.; Zwach, C.; di Primio, R.; Resumos do $20^{\text {th }}$ International Meeting on Organic Geochemistry, Nancy, France, 2001.

36. Wilhelms, A.; Larter, S. R.; Head, I.; Farrimond, P.; di Primio, R.; Zwach, C.; Nature 2001, 411, 1034.

37. Moldowan, J. M.; Lee, C. Y.; Sundararaman, P. Em Biological Markers in sediments and petroleum; Moldowan, J. M.; Albrecht, P.; Philp, R. P.; eds.; Prentice-Hall, Englewood Cliffs: New Jersey, 1992.

38. Walters, C. C. Em Encyclopedia of Geochemistry; Marshall, C. P.; Fairbridge, R. W.; eds., Kluwer Academic Publishers, Dorcrecht: the Netherlands, 1999.

39. Magot, M.; Ollivier, B.; Patel, B. K. C.; Antonie Leewenhock. 2000, 77, 103.

40. Cord-Ruwich, R.; Kleinitz, W.; Widdel, F.; J. Petrol. Technol. 1987, 1, 97. 
41. Orphan, V. J.; Taylor, L. T.; Hafenbradl, D.; Delong, E. F.; Appl. Environ. Microbiol. 2000, 66, 700 .

42. Bonch-Osmolovskaya, E. A.; Miroshnichenko, M. L.; Lebedinsky, A. V.; Chernyh, N. A.; Nazina, T. N.; Ivoilov, V. S.; Belyaev, S. S.; Boulygina, E. S.; Lysov, Y. P.; Perov, A. N.; Mirzabekov, A. D.; Hippe, H.; Stackebrandt, E.; L’Haridon, S.; Jeanthon, C.; Appl. Environ. Microbiol. 2003, 69, 6143 .

43. Borzenkov, I. A.; Milekhina, E. I.; Gotoeva, M. T.; Rozanova, E. P.; Beliaev, S. S.; Mikrobiologia 2006, 75, 82.

44. Coates, J. D.; Woodward, J.; Allen, J.; Philip, P.; Lovley, D. R.; Appl. Environ. Microbiol. 1997, 63, 3589.

45. Korda, A.; Santas, P.; Tenente, A.; Santas, R.; Appl. Environ. Microbiol. 1997, 48, 677

46. Da Cruz, G. F.; Santos Neto, E. V.; Marsaioli, A. J.; Org. Geochem. 2008, 39, 1204.

47. Röling, W. F. M.; Head, I. M.; Larter, S. R.; Res. Microbiol. 2003, 321.

48. Aitken, C. M.; Jones, D. M.; Larter, S. R.; Nature 2004, 431, 291.

49. Voordouw, G.; Armstrong, S. M.; Reimer, M. F.; Fouts, B.; Telang, A. J.; Shen, Y.; Gevertz, D.; Appl. Environ. Microbiol. 1996, 62, 1623.

50. Azevedo, D. de A.; da Silva, T. F.; da Silva, D. B.; Quim. Nova 2009, 32, 1770.

51. Liao, Y.; Geng, A.; Huang, H.; Org. Geochem. 2009, 40, 312.

52. Atlas, R. M.; Mar. Pollut. Bull. 1995, 31, 178.

53. Atlas, R. M.; Microbiol. Rev. 1981, 45, 180.

54. Wentzel, A.; Ellingsen, T. E.; Kotlar, H-K.; Zotchev, S. B.; Throne-Holst, M.; Appl. Microbiol. Biotechnol. 2007, 76, 1209.

55. Evans, P. J.; Ling, W.; Goldschmidt, B.; Ritter, E. R.; Young, L. Y.; Appl. Environ. Microbiol. 1992, 58, 496.

56. Berthe-Corti, L.; Höpner, T.; Paleobiology 2005, 219, 171.

57. Fritsche, W.; Hofrichter, M. Em Biotechnology; Klein, J., ed.; John Wiley \& Sons: New York, 2000.

58. Vazoller, R. F.; Tese de Doutorado, Universidade de São Paulo, Brasil, 1995.

59. Aeckersberg, F.; Bak, F.; Widdel, F.; Arch. Microbiol. 1991, 156, 5.

60. So, C. M.; Phelps, C. D.; Young, L. Y.; Appl. Environ. Microbiol. 2003, 69, 3892.

61. Cravo-Laureau, C.; Grossi, V.; Raphael, D.; Matheron, R.; HirschlerRéa, A.; Appl. Environ. Microbiol. 2005, 71, 3458.

62. Ehrenreich, P.; Behrends, A.; Harder, J.; Widdel, F.; Arch. Microbiol. 2000, $173,58$.

63. Zengler, K.; Heider, J.; Roselló-Mora, R.; Widdel, F.; Arch. Microbiol. 1999, 172, 204.

64. Widdel, F.; Rabus, R.; Curr. Opin. Biotechnol. 2001, 12, 259.

65. Wilkes, H.; Rabus, R.; Fischer, T.; Armstroff, A.; Behrends, A.; Widdel, F.; Arch. Microbiol. 2002, 177, 235.

66. Wilkes, H.; Behrends, A.; Armstroff, A.; Fischer, T.; Pierik, A. J.; Widdel, F.; J. Bacteriol. 2001, 183, 1707.
67. Kropp, K. G.; Davidova, I. A.; Suflita, J. M.; Appl. Environ. Microbiol. 2000, 66, 5393

68. Grossi, V.; Cravo-Laureau, C.; Guyoneaud, R.; Ranchou-Peyruse, A.; Hirschler-Réa, A.; Org. Geochem. 2008, 39, 1197.

69. Bost, F. D.; Frontera-Suau, R.; McDonald, T. J.; Peters, K. E.; Morris, P. J.; Org. Geochem. 2001, 32, 105.

70. Alexander, R.; Kagi, R. I.; Woodhouse, G. W.; Volkman, J. K.; Aust. Petrol. Explor. Assoc. J. 1983, 23, 53.

71. Connan, J.; Restle, A.; Albrecht, P.; Adv. Org. Geochem. 1980, 12, 1.

72. Seifert, W. K.; Moldowan, J. M.; Geoch. Cosmochim. Acta 1979, 43, 111.

73. Wang, Z.; Fingas, M. F.; Sigouin, L.; Owens, E. H.; Proceedings International Oil Spill Conference, Tampa, USA, 2001, p. 115.

74. Orphan, V. J.; Taylor, L. T.; Hafenbradl, D.; Delong, E. F.; Appl. Environ. Microbiol. 2000, 66, 700.

75. Chaillan, F.; Flèche, A.; Bury, E.; Phantavong, Y.; Grimont, P.; Saliot, A.; Oudot, J.; Res. Microbiol. 2004, 155, 587.

76. De Vasconcellos, S. P.; Crespim, E.; Da Cruz, G. F.; Marques-Simioni, K. C.; Santos Neto, E. V.; Marsaioli, A. J.; Oliveira, V. M.; Org. Geochem. 2009, 40, 574.

77. Toledo, L. G.; Calvo, C.; Rodelas, B.; González-López, J.; J. Syst. Appl. Microbiol. 2006, 29, 244

78. DuTeau, N. M.; Rogers, J. D.; Bartholomay, C. T.; Reardon, K. F.; Appl. Environ. Microbiol. 1998, 64, 4994.

79. Da Silva, A. A.; Tese de Doutorado, Universidade Estadual de Campinas, Brasil, 2008.

80. De Lima, S. G.; Steffen, R. A.; Reis, F. de A. M.; Koike, L.; Santos Neto, E. V.; Cerqueira, J. R.; Lopes, J. A. D.; Org. Geochem. 2010, 41, 325.

81. Koike, L.; Rebouças, L. M. C.; Reis, F. de A. M.; Marsaioli, A. J.; Org. Geochem. 1992, 18, 851 .

82. Yen, T.-W.; Marsh, W. P.; Mackinnon, M. D.; Fedorak, P. M.; J. Chromatogr., A 2004, 83, 1033.

83. Scott, A. C.; Younge, R. F.; Fedorak, P. M.; Chemosphere 2008, 73, 1258.

84. Rodrigues, R.; Brüning, I. M. R. A.; Boletim Técnico da Petrobras 1984, 27,3 .

85. Brooks, P. W.; Fowler, M. G.; MacQueen, R. W.; Org. Geochem. 1988, $12,519$.

86. Peters, K. E.; Moldowan, J. M.; Org. Geochem. 1991, 17, 47.

87. Thorn, K. A.; Aiken, G. R.; Org. Geochem. 1998, 29, 909

88. Taylor, P. N.; Bennett, B.; Jones, D. M.; Larter, S. R.; Org. Geochem. 2001, 32, 341 .

89. Wenger, L. M.; Davis, C. L.; Isaksen, G. H.; SPE 71450, Society of Petroleum Engineers, 2001, 375. 